\title{
POLIFEMO, GALATEA E LE ORIGINI DI ILLIRI E CELTI ALLA LUCE DELLE FONTI ANTICHE (TIMEO, APPIANO) E UMANISTICHE (FR. FILELFO, N. COMES)
}

\begin{abstract}
According to Appian of Alexandria (Illyr. 2.3-4), Sicilian Cyclops Polyphemus and his beloved sea-nymph Galatea are identified as parents of Illyrius, Celtus and Galas, progenitors of the Illyrians, the Celts, and the Galatians. Polyphemus' children all migrated from Sicily and ruled over the peoples named after them. Appian echoes a fragment of Timaios of Tauromenion (FGrH 566 F69). This genealogical aition is linked with Dionysius I' expansionism in the Balkans, in order to prevail in Adriatic and Ionian seas by establishing strategic alliances with local Illyrians and Celts. Myths relating to the eponymous ancestors of Illyrian and Celtic peoples originated in Syracuse's court and were most likely composed to support the political aims of the tyrant. Finally, Italian humanist Fr. Filelfo also chronicles the genealogical legend of Polyphemus in a letter of 1471, with respect to the Crusade against the Turks and 16th century mythographer Natalis Comes also quotes this story.
\end{abstract}

Key words. - genealogy, ethnography, Dionysius I of Syracuse, Polyphemus, Illyrians, Celts, Greek colonization, humanism.

\section{Premessa}

Il mito di Galatea e Polifemo e della progenie nata dalla loro unione offre al tiranno Dionisio I (431/0-368/7 a.C. $)^{1}$ un tema propagandistico di notevole risonanza di rimando all'intesa di Siracusa con i Celti e gli Illiri. Secondo una tradizione risalente a Timeo di Tauromenio (356-260 a.C. ca.), rilanciata da Appiano di Alessandria (95-165 ca.), le nozze tra il Ciclope siciliano e la sua amata nereide sono coronate dalla nascita di tre figli: Celto, Illirio e Gala(ta), i quali partono dall'Isola per regnare su Celti, Illiri e Galati e diventarne gli eponimi. ${ }^{2}$

${ }^{1}$ Per il contenuto formale del potere di Dionisio I che adombra la regalità, pur in assenza del titolo esplicito di basileus, cfr. De Vido 2013.

${ }^{2}$ Cfr. Coppola 1993a, 17; Ead. 1995, 92; Ead. 2000, 12; Briquel 1995, 243-245; Alessandrì 1997, 139-140; Sordi 1999, 109-116 = Ead. 2002, 577-585; Bearzot 2004, 6364; Muccioli 2004, 134; Castiglioni 2014, 25; De Vido 2015, 185; Troiani 2018, 153. 


\section{Fonti antiche}

Per l'analisi di questa leggenda genealogica si rileva una discussione in dettaglio nell' 'T $\lambda \nu \rho \imath \kappa \dot{\eta}$, il resoconto sulle conquiste romane nell'Adriatico orientale e nei Balcani occidentali, che Appiano colloca

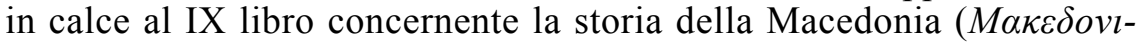
$\kappa \eta$ ), ma i copisti bizantini hanno aggregato al V libro dei Bella Civilia. ${ }^{3}$ Il profilo etnografico delineato dallo storico di età imperiale contiene un'interessante "archeologia" sulle origini degli Illiri: ${ }^{4}$

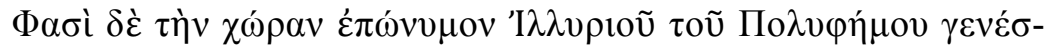

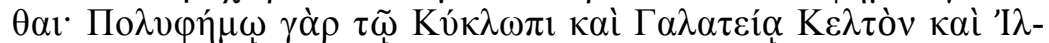

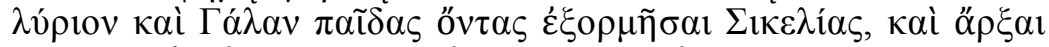

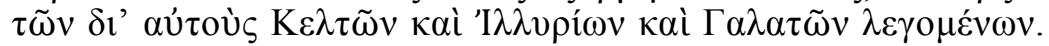

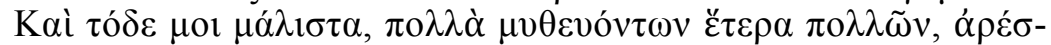
$\kappa \varepsilon 1$.

Si narra che la regione dell'Illirico prese nome da Illirio, figlio di Polifemo. Il ciclope Polifemo e Galatea ebbero come figli Celto, Illirio e Gala. Questi salparono dalla Sicilia e regnarono sui popoli che da loro furono denominati Celti, Illiri e Galati. E questa è per me sicuramente la versione preferibile, giacché circolano molte altre storie su questo soggetto.

Come affermato esplicitamente, Appiano opera una cernita tra una pluralità di racconti inerenti alle origini mitiche di Illiri e Celti diffusi da altri autori $(\mu v \theta \varepsilon v o ́ v \tau \omega \nu \ldots \pi \mathrm{o} \lambda \lambda \tilde{\omega} v$, con poliptoto enfatico per quest'inciso: $\pi \mathrm{o} \lambda \lambda \dot{\alpha} \ldots \pi \mathrm{o} \lambda \lambda \tilde{\omega} v)$. Si tratta di popoli geograficamente distanti e dissimili, entrati ben presto nella sfera d'interazione dell'ecumene greco-romana. Di seguito, lo storico alessandrino elenca i figli di Illirio: Encheleo, Autarieo, Dardano, Medo, Taulante, Perrebo e altri divenuti eponimi di genti insediate nei Balcani. ${ }^{5}$ Infine, Autarieo è menzionato come padre di Pannonio (o Peone), il quale generò

${ }^{3}$ Cfr. Šašel Kos 2004, 141-142, 146 per la valutazione dell'Illyriké nel complesso dell'opera storiografica di Appiano, il quale non si propone di scrivere una storia sistematica dei Balcani anche per mancanza di fonti sull'argomento, come lamenta espressamente (Illyr. 6.14.29) e attinge a informazioni disparate: questa è l'unica opera pervenutaci dedicata alla storia della regione ed è preziosa, malgrado brevità e asimmetrie, cfr. Gabba 1971, 187; Dzino 2010, 8; Goukowski 2011, 3; Castiglioni 2014, 25.

${ }^{4}$ Appian, Illyr. 2.3 = Hist. Rom. 10.2.3 per la leggenda della discendenza di Illiri, Galati e Celti dal $\gamma$ ó $\mu$ os tra Polifemo e Galatea.

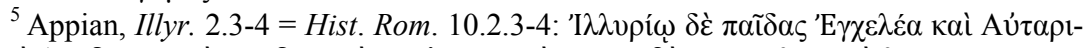

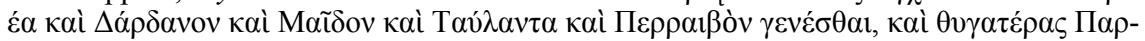

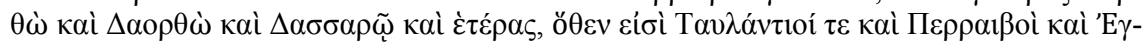

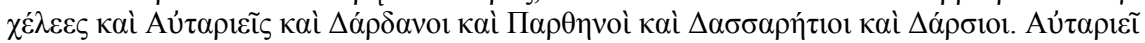

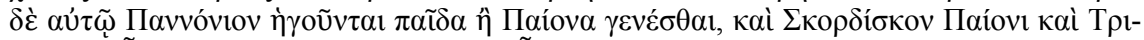

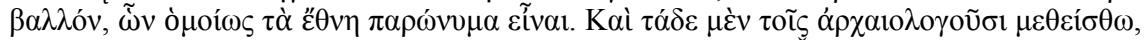

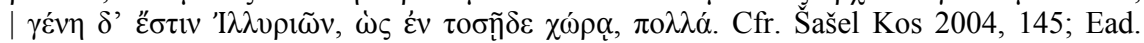
2005, 115-117 per la conclusione del catalogo imputabile ad un'aggiunta seriore. Sui popoli illirici meridionali (Ardiei, Daorsi, Taulanti), cfr. Dzino 2010, 38. 
Scordisco e Triballo, altri capostipiti di tribù balcaniche ${ }^{6}$ Per l'Illirico bisogna intendere una vasta area delimitata a ovest dall'Adriatico ed estesa fino al Danubio, i cui confini orientali sono segnati approssimativamente dai fiumi Drava a Nord, Morava e Vardar a Sud. ${ }^{7}$ Nel complesso, il quadro desumibile dal prospetto etnografico di Appiano si riferisce alla visione dell'Illirico acquisita con il consolidamento della conquista romana grazie alle spedizioni di Ottaviano e Tiberio in un'età posteriore rispetto alla nascita del nucleo primigenio della leggenda impostata nell'età di Dionisio il Vecchio e ampliata in seguito con altri eponimi balcanici. ${ }^{8}$

La notizia di Appiano mostra evidenti affinità con un frammento di Timeo tramandato dall'Etymologicum Magnum, che è ipotizzabile come la fonte diretta o indiretta dell' Illyriké ${ }^{-9}$ ed è pervenuto nella seguente forma, in cui compare un solo figlio, Galato: ${ }^{10}$

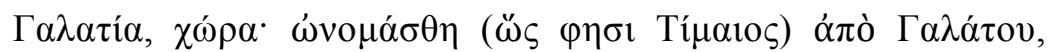

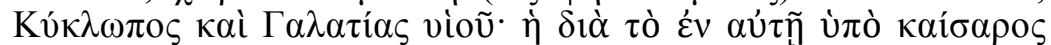

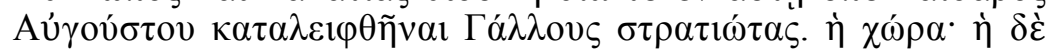
$\pi$ ó $1 \varsigma$ "А $\gamma \kappa v \rho \alpha$.

Galazia, regione, fu chiamata così (come dice Timeo) da Galato, figlio del Ciclope e di Galatea, Al tempo dell'imperatore Augusto fu occupata da soldati celti. La città è Ancyra.

Nei figli di Polifemo si riconoscono ragionevolmente i progenitori di Celti e Illiri stanziati nell'Adriatico orientale e dei Galli d'Italia

\footnotetext{
${ }^{6}$ Sulle tribù ricordate in quest'excursus riferito all'età di Filippo II e Alessandro, cfr. Ferrone 2004, 37-38 e n. 30. In prospettiva storica le fonti medio-bizantine assegnano il titolo di T $\rho ı \alpha \lambda \lambda$ oí a tribù protoserbe, cfr. Joh. Scylitzes, Thurn 424 di rimando a Stefan Vojislav (1018-43), signore di Duklja (greco $\Delta$ ıók $\lambda \varepsilon 1 \alpha$ ) nell'attuale Montenegro. Con l'ascesa della dinastia dei Nemanjić (1166-1371) l'etnonimo è identificato con "Serbi" ( $\Sigma \varepsilon ́ \rho-$

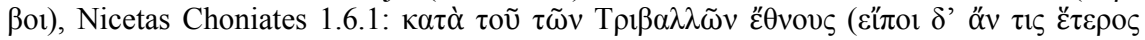
$\Sigma \dot{\varepsilon} \rho \beta \omega v)$, cfr. Malamut 1998, 450-451; Pontani 2017, 492-493 n. 79.

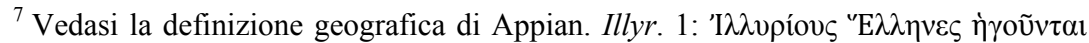
$\tau$ où

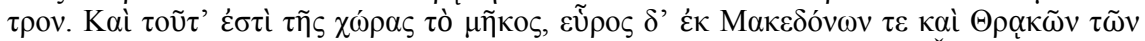

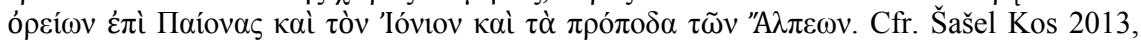
3408: in questa descrizione, come in Strabone (7.5.3 C 314) e Cassio Dione (12 Zonar. 8.19.8), l'Illirico corrisponde ai Balcani nordoccidentali compresi tra il Mar Adriatico fino al golfo Rizonico (Bocche di Cattaro) e la Pannonia.

${ }^{8}$ Cfr. Šašel Kos 2005, 115-117; Dzino 2010, 56; Castiglioni 2014, 25 con rinvio a Suet. Tib. 16.4 (a. 12 d.C.).

${ }^{9}$ Sulla vexata quaestio delle fonti di Appiano, cfr. in genere Hahn 1982; Westall 2013, Id. 2015, 140 per l'ammissione specifica di avere consultato fonti sulle campagne illiriche del I secolo a.C.

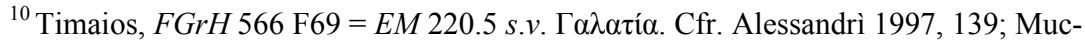
cioli 2001, 355. Come suggerisce anche Briquel 1995, 243 non è azzardato ipotizzare una genealogia più complessa, simile a quella tràdita in Appiano, inclusiva di Celto e Illirio a fianco dell'eponimo dei Galati.
} 
venuti a contatto diretto con i Greci. ${ }^{11}$ Illirio è menzionato anche in uno scolio virgiliano, che tramanda la medesima genealogia. ${ }^{12}$

La localizzazione siciliana della saga è ignota a Omero, il quale omette riferimenti geografici precisi al riguardo. ${ }^{13}$ Le sedi dei Ciclopi sono fissate presso l'Etna nella tradizione acquisita dal siracusano Epicarmo, il quale mostra diversi punti di contatto con il modello odissiaco, ma lo risemantizza in modo autonomo, presentando un personaggio culturalmente più progredito che esibisce doti magiriche, poetiche e musicali. ${ }^{14}$ L'ambientazione in Sicilia è recepita, quindi, da Tucidide ed Euripide, ${ }^{15}$ che conferisce al Ciclope doti desunte dall'ethos aristocratico arcaico. ${ }^{16}$

La costruzione ideologica, che postula legami genealogici tra $\mathrm{i}$ popoli celtici e illirici, solletica indubbiamente le velleità espansionistiche di Dionisio il Vecchio nell'Adriatico. ${ }^{17}$ Sulle due sponde di questo bacino l'attività colonizzatrice siracusana è documentata fin dal $390 / 88 .{ }^{18}$ Nel progetto siracusano mirante a contrastare la pirateria e

11 La Nereide Galatea è riconnessa anche per assonanza fonetica ai Galli e all'eponimo Galata, mentre il richiamo ai Celti s'instaura soltanto a partire dal figlio Celto, cfr. Anello 1980, 68-70; Braccesi 1991a, 96; Briquel 1995, 242; Alessandrì 1997, 140; Vaglio 2001, 174.

${ }^{12}$ Schol. Verg. Ecl. 8.7: Illyri ... nomen habent ab Illyrio Polyphemi filio, qui genitus fuit et Cyclope et Galatea, ut ait Apronius, cfr. Briquel 1995, 243.

${ }^{13}$ L'Od. 9.187-542, in part. 196-215 offre il prototipo del mostro antropofago, un $\alpha^{\prime} \gamma$ -

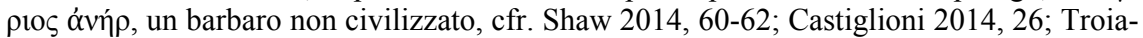
ni 2018, 135.

${ }^{14}$ Il Ciclope di Epicarmo (fr. 70-72 K.-A.) sanziona l'ambientazione siciliana e l'evoluzione del personaggio ingentilito, confermata dagli altri drammi satireschi di Euripide ed Aristia ( $f r .4$ Sn.) e dalle attestazioni comiche di Cratino, Odissei ( $f r$. 143-157 K.A.), Callia, Ciclopi (fr. 5-13 K.-A.), cfr. Sutton 1983; Mastromarco 1998, 11-26, 34; Shaw 2014, 62-65; Castiglioni 2014, 26; Lelli 2017, 218; Troiani 2018, 135-136.

${ }^{15}$ Th. 6.2.1 per Ciclopi e Lestrigoni situati in Sicilia; il dramma satiresco euripideo è ambientato ai piedi dell'Etna, la vetta più elevata dell'Isola (Cycl. 114: Aícvoĩos, ő $\chi 0$ os $\Sigma 1 \kappa \varepsilon \lambda i ́ \alpha \varsigma$ ù $\pi \varepsilon ́ \rho \tau \alpha \tau o \varsigma)$, cfr. Cusset 2004, 26, 29: il Ciclope con il suo unico occhio offre una rappresentazione simbolica del cratere vulcanico; Castiglioni 2014, 26; Troiani 2018, 142.

${ }^{16}$ Il Polifemo euripideo non è digiuno di storia e letteratura, s'interessa alle vicende del conflitto troiano (275-284), declama versi lirici seppure storpiati (323-331), inoltre, aderisce al modello pederastico (582-589), cfr. Burzacchini 1979; Mastromarco 1998, 3233; Troiani 2018, 136-137.

${ }^{17}$ Cfr. Anello 1980, 69-72; Ead. 1996, 406; Ead. 1999, 142-145; Sordi 1999, 109; Uggeri 2002, 295; Pierozzi 2019, 66: il mito di Polifemo e Galatea risponde all'esigenza della legittimazione della politica di espansione europea con evidenti aperture filo-barbariche. Sulla centralità dello spazio ionico-adriatico nella politica di Dionisio I, cfr. Braccesi 1991b; De Vido 2015, 177.

${ }^{18}$ Il mito di Polifemo e Galatea si collega agli esordi dell'attività coloniaria di Dionisio il Vecchio, la cui cronologia concerne l'esegesi del racconto diodoreo: 15.3.4-5, cfr. Anello 1980, 66-72; Ead. 1996, 406; Vanotti 1991, 107; Alessandrì 1997, 139; De Sensi Sestito 2011, 362, certo essa s'infittisce negli anni ' 80 in concomitanza di un periodo di quiete sul fronte dello scontro greco-punico con un'accentuata presenza celtica nell'Italia centrosettentrionale, cfr. Pierozzi 2019, 45, 59, 61. 
assicurare la sicurezza alla navigazione commerciale in tale area ${ }^{19}$ rientra la fondazione di Issa nell'isola di Lissa (Viš), che costituisce a sua volta le subcolonie sulla costa dalmata di Trag(o)urion, odierna Traù (Trogir) ed Epetion, oggi Stobrezio (Stobreč) presso Spalato (Split), poco a nord di Salona. Sotto l'egida di Dionisio I avviene pure la colonizzazione paria di Pharos, odierna Cittavecchia (Stari Grad) nell'isola di Lesina (Hvar). ${ }^{20}$ Issa fonda, inoltre, un insediamento presso il villaggio di Lombarda (Lumbarda) nell'isola di Curzola (Korčula), dove si segnala la colonia cnidia di Kerkyra Melaina (Corcyra Nigra). ${ }^{21}$ Il tiranno si allea notoriamente agli Illiri nello scacchiere epirota e tenta con il loro aiuto d'insediare sul trono il molosso Alceta, accolto come ospite alla corte siracusana durante il suo esilio d'incerta datazione. ${ }^{22}$ Sul piano storico-religioso la connessione con l'Epiro è suffragata dal sacerdozio dei Galeoti, rinomati indovini siculi, il cui capostipite Galeote di ascendenza iperborea parte, secondo la leggenda, da questa regione della Grecia occidentale dopo aver consultato l'oracolo di Dodona, ${ }^{23}$ che lo invia in Sicilia a svolgere l'attività d'interprete di sogni e prodigi trasmessa ai suoi discendenti. ${ }^{24}$

Inoltre, i Siracusani promuovono vigorosamente l'ellenizzazione degli indigeni. Si rivela illuminante, a tal riguardo, il dibattito a pro-

${ }^{19}$ Gli Illiri svolgono un ruolo decisivo come agenti del commercio, marineria e pirateria nell'Adriatico e nel Canale d'Otranto, cfr. Ferone 2004a; Id. 2004b, 37-38 e n. 30 di rimando ai dati di Appian. Illyr. 3; Lombardo 2004, 52, 55; Intrieri 2015, 70, per il concorso di Iapigi e Peuceti a tali attività, ibid. 74 .

${ }^{20}$ Cfr. Diod. 15.13.4-14; ps.-Scymnus 413-414 per l'isola di Lissa sede della colonia

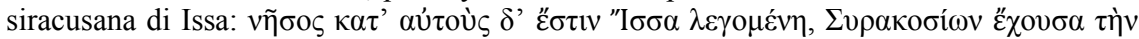

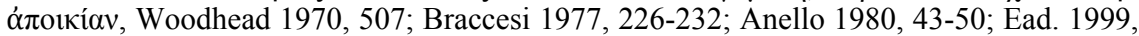
117-119; Rendić-Miočević 1983, 189-191; Coppola 1988, 223; Ead. 1993a, 18-19; Mastrocinque 1988, 36; Caven 1990, 182-183; Coppola 2002, 373; Lombardo 2002, 428-429, 435; Id. 2004, 52; Cabanes 2008, 175-177; Castiglioni 2014, 30; Intrieri 2015, 74; Pierozzi $2019,59-60$.

${ }^{21}$ Cfr. ps.-Scymnus 426-430; Mastrocinque 1988, 7-9; Intrieri 2015, 75.

${ }^{22}$ La symmachía di Dionisio I con gli Illiri si colloca verso il 385 in funzione antispartana (Diod. 15.13.2-3), si ricordi l'attitudine filoateniese del Molosso, cfr. Vanotti 1996, 78-80; Sordi 1999, 112-115; Ead. 2000a, 67; De Sensi Sestito 2011, 361-364 per i rapporti tra Alceta e Dionisio; Castiglioni 2014, 29; Intrieri 2015, 75-77; Pierozzi 2019, 61 retrodata l'intesa e l'avventura adriatica siracusana.

${ }^{23}$ Per questa famiglia mantica Steph. Byz. s.v. $\Gamma \alpha \lambda \varepsilon \tilde{\omega} \tau \alpha \iota$ connette espressamente il nome di questi indovini al geco ( $\gamma \alpha \lambda \varepsilon \hat{\varsigma} \varsigma \delta \dot{\varepsilon} \kappa \alpha \grave{~ o ̀ ~} \alpha \sigma \kappa \alpha \lambda \alpha \beta \omega ́ \tau \eta \zeta)$, animale ctonio sacro ad Apollo, Signore della mantica, per l'ascalabotomanzia, cfr. Waegeman 1984, 219-223; Costanza 2009, 45. L'oracolo di Dodona invia Galeote verso Occidente in Sicilia e Telmesso in Caria per fondare un altro reputato centro di oniromanzia, cfr. Mastrocinque 1980-81; Marotta 2003.

${ }^{24}$ La tradizione sui Galeoti, interpreti siciliani di portenti consultati anche per la nascita di Dionisio I, è riportata da Paus. 5.23.6-7, Cic. Div. 1.39 e deriva recta via dall' autorità di Filisto (FGrH 556 F57a, b, F58), cfr. Braccesi 1991a, p. 93; Sordi 1999, 109; Moscati Castelnuovo 2014, 17, 21, con la proposta di riconoscere l'elaborazione della leggenda da parte di uno storico epirota come Prosseno vicino a Pirro, autore di una storia lo-

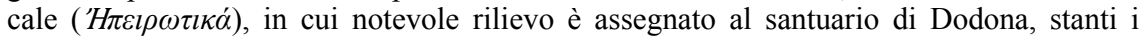
frammenti superstiti. 
posito dell'etnicità degli Illiri collocabili nel promontorio tra Spalato e Sebenico (Šibenik), Illiri della terraferma, i quali combattono contro le prospicienti colonie siracusane, ma sono profondamente grecizzati, ${ }^{25}$ come sottolinea una solida tradizione riconducibile ad uno storico ostile alla tirannide quale Timeo. ${ }^{26}$

Alla luce dei sottintesi politici conviene ripercorrere il tema etnogonico correlato a Polifemo che è abilmente sfruttato dalla Corte dionigiana, in linea con aspirazioni politiche cogenti e amicizie interessate nei riguardi di popoli allogeni accortamente utilizzati come mercenari nel quadro di un'intesa militare chiaramente stabilita. Il vincolo di mercenariato rinsalda relazioni stabili intestate all'iniziativa di Dionisio il Vecchio, il quale determina sensibili aperture a elementi celtici $^{27}$ e illirici, ${ }^{28}$ impostando una linea politica ereditata dai dinasti siracusani. $^{29}$

La filiazione da Polifemo connota i popoli storicamente attestati nello spazio danubiano e balcanico come particolarmente bellicosi e intemperanti. La diffusione di questo topos etnogonico è imputabile alla propaganda di Dionisio I: il tema della tradizione timaica s'inserisce perfettamente nel disegno strategico di promuovere l'alleanza tra

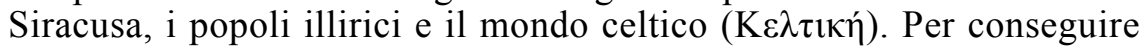
tale finalità è rilevante l'ascendenza comune dei popoli sopra menzionati dal Ciclope siciliano. ${ }^{30}$ La propaganda di matrice siracusana attira nell'alveo della grecità queste popolazioni con il duplice risultato di sottrarle agli orizzonti di un'alterità barbarica e rivendicare nello stesso tempo ai Sicelioti un primato indiscusso, in quanto mitici capostipiti collocati alle origini della storia dell'Occidente in prospettiva rigorosa-

${ }^{25}$ Sul carattere ellenico degli Illi e le loro presunte origini doriche a partire da Illo, figlio di Eracle, cfr. ps.-Scymnus 408-412 = Tim. FGrH 566 F77; Erat. fr. III B, 113.356 Berger, ps.-Scylax 22, Mastrocinque 1988, 38-40, con ulteriori considerazioni sull'ellenizzazione degli Illiri e le varianti della propaganda sullo scacchiere adriatico; D'Andria 2002, 120; Lombardo 2002, 436-437; Castiglioni 2014, 30; Intrieri 2015, 76.

${ }^{26}$ Sulla condanna timaica della tirannide, cfr. Vattuone 1991, 131-141.

${ }^{27}$ Nelle forze ausiliarie inviate da Dionisio I agli Spartani nel conflitto contro Tebe (369/8) si annoverano elementi celtici impiegati in seguito nelle guerre contro Cartagine e pure da Agatocle nell'invasione del Nordafrica (310/7), cfr. Maier 2012, 45; Castiglioni 2014, 29; Pierozzi 2019, 58. Sull'interesse celtico di Dionisio I, cfr. Coppola 2000, 12.

${ }^{28} \mathrm{~L}$ 'apertura ai dinasti illirici assicura il sostegno di truppe mercenarie, favorendo la penetrazione del commercio siracusano nel Nord Adriatico che è alla base della genealogia dei tre eponimi, cfr. Braccesi 1991a, 91-92; Id. 1994, 95-102; Briquel 1995, 244; Vanotti 1996, 82; Rossignoli 2004, 117-118; Intrieri 2015, 78.

${ }^{29}$ Sull'attitudine di Agatocle verso la pirateria adriatica e ionica, con intervento a Corcira, concessa come dote alla figlia Lanassa data in sposa a Pirro (295) e poi a Demetrio Poliorcete (291/0), e nelle isole vicine di Leucade, Itaca, cfr. Consolo Langher 2002; Lombardo 2004, 56-57; De Sensi Sestito 2011, 367-369; Antonetti 2015, 200-202; De Vido 2015, 180-182.

${ }^{30}$ Cfr. Anello 1980, 68-70; Ead. 1984, 11-51; Braccesi 1994, 95-110; Rossignoli 2004, 118; Pierozzi 2019, 65 con la definizione di «rigorosa macchinazione propagandistica cortigiana». 
mente ellenocentrica. ${ }^{31}$ Di converso, sul fronte interno Dionisio sfrutta costantemente la paura del pericolo cartaginese per eliminare l'opposizione oligarchica e rafforzare il suo potere, accreditandosi come il campione dell'Ellenismo isolano: grazie alla sua aura di condottiero vittorioso, favorito dalla Tyche, ${ }^{32}$ respinge la minaccia punica e dilata la sua azione a largo raggio nel Mediterraneo orientale e nei Balcani occidentali. ${ }^{33}$

Fin dal principio del IV secolo a.C. i Celti esercitano a loro volta una pressione costante sull' area danubiana e le regioni illiriche e interagiscono in modo significativo con i popoli balcanici, delineando un quadro etnico-politico articolato. ${ }^{34}$ Già dagli esordi del regno di Alessandro Magno i Celti figurano nel ruolo di alleati dei Macedoni contro gli Illiri. ${ }^{35}$ È nota l'ambasciata inoltrata dai Celti dell'Adriatico ad Alessandro, mentre questi si trova in Tracia sul Danubio (primavera 335) per domare una rivolta di tribù locali, illiriche e dei Triballi, in attesa di partire per la sua spedizione asiatica. In tale occasione i "barbari" recano proposte di amicizia e impersonano un paradigma di $\grave{\alpha} \pi-$ $\lambda o ́ \tau \eta \zeta$, dichiarando al sovrano macedone di abitare in una regione impervia, di difficile accesso e non temere nulla eccetto che il cielo cada sulle loro teste. ${ }^{36}$ La provenienza di questa legazione è stata, plausibilmente, individuata nei Celti giunti dalle due colonie fondate da Dionisio II (397-343 a.C.) in Apulia tra il 367 e il 359 per proteggere il commercio nel Canale d'Otranto, piuttosto che a Celti balcanici inse-

\footnotetext{
${ }^{31}$ Sulla "cattura" dell'elemento indigeno attraverso strumenti elaborati dalla propaganda dionigiana, cfr. Coppola 1991, 103-106; Ead. 1993a, 17; Muccioli 2001, 355; Pierozzi 2019, 62-63. Per la caratterizzazione affettiva che rivendica alle tradizioni siciliane la figura del Ciclope percepito come un conterraneo, cfr. Lelli 2017, 217, 266.

32 Tale immagine celebrativa del tiranno è collegata al suo ruolo di protetto della dea poliade Syra/Sosia, la quale è raffigurata sul recto dei bronzi dionigiani con legenda $\Sigma Y$ PA e ha funzione di Tyche Salvatrice, svolgendo un ruolo palingenetico, cfr. Caccamo Caltabiano 2002, 40, 44-45.

${ }^{33}$ L'ascesa del tiranno segue la travolgente avanzata cartaginese con la presa di Imera (409) e Agrigento (406) e si consolida grazie alla fama di comandante vittorioso, cfr. Merante 1972-1973, 99-102; Sordi 1979, 212, 217; Ead. 1980, 23; Scarpa Bonazza Buora 1984, 13-14, 39; Caven 1990, 149; Consolo Langher 1997, 102; Occhipinti 2006, 65-67; Intrieri 2015, 68.

${ }^{34}$ Per la presenza celtica nei Carpazi e l'invasione della Pannonia, di cui riferisce un estratto di Pompeo Trogo in Giustino (XXIV 4), cfr. Szabó 1988, 13-15; Id. 2007, 5, 7-10 con riconsiderazione dei documenti archeologici; Krutas 2000, 89, 232; Bearzot 2004, 63.

${ }^{35}$ Cfr. Strabo 7.3.8, Arrian. Anab. 1.4.6-8; Alessandrì 1997, 131; Urso 2001, 25; Demandt 1998, 23, con osservazioni sulla formula di giuramento di tale alleanza conforme alle consuetudini tramandatesi nell'Irlanda gaelica medievale.

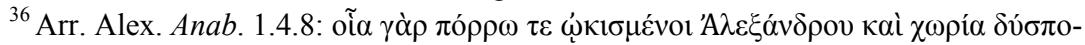

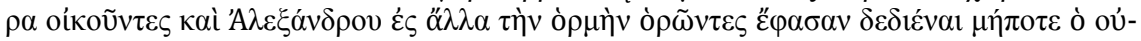

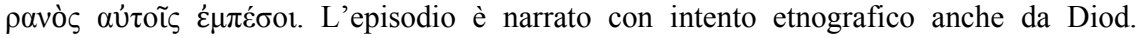
17.113.2, cfr. Sordi 1985, 212, con rinvio a Tolomeo come fonte della storia; Alessandrì 1997, 132-133; Santamato 2013, 247.
} 
diati a nord e a est dell'Illirico. ${ }^{37}$ Questi elementi sorvegliano la navigazione nel Basso Adriatico e discendono dai Galli insediatisi in Italia dopo la presa di Roma e assoldati varie volte come mercenari dai due Dionigi in funzione antietrusca e antiromana. ${ }^{38}$

L'alleanza con i mercenari celti si è rivelata cruciale per Dionisio il Vecchio, nonostante la naturale apprensione nei confronti di questi guerrieri temibili, i quali minacciano anche molte città greche in Italia con le loro irruzioni nella penisola. Nondimeno, quest'intesa è parsa una soluzione opportuna al pari dell'attitudine filo-illirica. Tale politica ha alimentato la leggenda nobilitante atta a giustificare i legami reciproci di interazione e mutua convenienza tramite il yó $\mu \circ \varsigma$ di Polifemo e Galatea. ${ }^{39}$

Appiano preferisce evidentemente per Illirio la genealogia siciliana in base ad una scelta consapevole, come espressamente rileva ( $\tau$ ó-

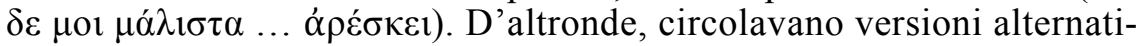
ve con rinvio ad antenati desunti ugualmente dai primordi del mito greco. Lo ps.-Apollodoro fa riferimento alla saga tebana, ${ }^{40}$ indicando Cadmo e Armonia quali genitori dell'eroe eponimo prima della loro metamorfosi ofitica, premessa della definitiva apoteosi: ${ }^{41}$

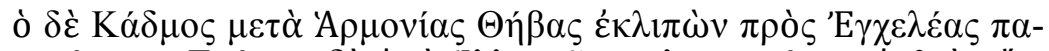

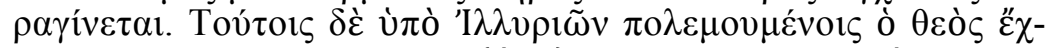

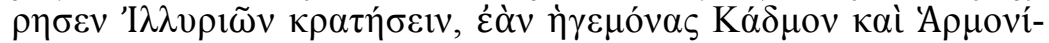

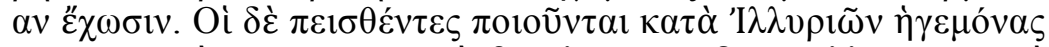

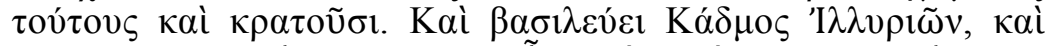

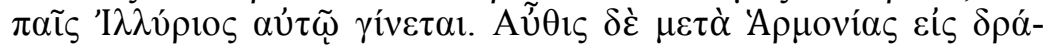

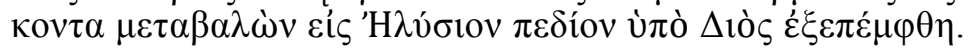

Cadmo lascia Tebe insieme con Armonia e si reca presso gli Enchelei. Costoro erano continuamente provocati alla guerra dagli Illiri e il dio aveva profetizzato che li avrebbero vinti se avessero scelto come capi Cadmo e Armonia. Essi obbediscono, fanno di Cadmo e Armonia i loro capi contro gli Illiri e vincono. Cadmo

${ }^{37}$ Proposta di Sordi 1981-82, 5; Ead. 1983, 14-16; concordano Alessandrì 1997, 134, 138; Urso 2001, 25. Sulla fondazione di Dionisio il Giovane, cfr. Diod. 16.5.3 e 10.2, con analisi di Lombardo 2004, 52-54.

${ }^{38}$ Tale vincolo di mercenariato è correlato a un rapporto di societas/amicitia tra i Galli e Siracusa all'indomani della caduta di Roma (lug. 387), cfr. Sinatra 1996; Castiglione 2014, 29; Pierozzi 2019, 57-58.

${ }^{39}$ Cfr. Coppola 1991, 103-106; Ead. 1993b, 110; Vanotti 1993, 120; Briquel 1995, 244; Muccioli 2004, 140; De Vido 2015, 185; Troiani 2018, 153.

${ }^{40} \mathrm{Cfr}$. Livadiotti 2012, 175.

${ }^{41}$ Ps.-Apollod. Bibl. 3.5.4 (39), trad. Ciani in Scarpi 1996, 209, 211; cfr. Eur. Ba. 1330-39, Nonn. D. 44.115-118; Cioppa 1993, 209; Šašel Kos 1993, 115-117, 122. Cfr. an-

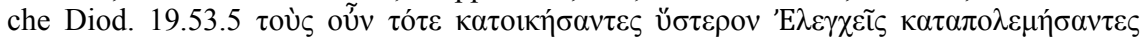

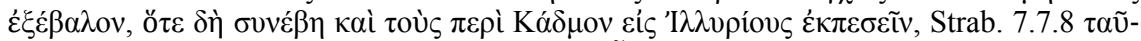

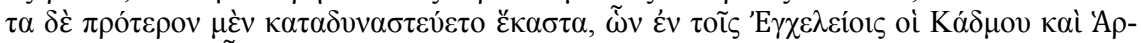

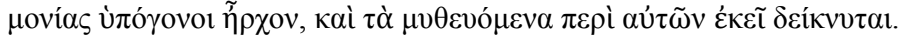


diventa re degli Illiri e gli nasce un figlio, Illirio. Più tardi egli e Armonia, trasformati in serpenti, furono mandati da Zeus nei Campi Elisi.

Secondo questa tradizione veicolata dai Corinzi nell'Alto Adriatico Cadmo avrebbe condotto la guerra contro gli Illiri dissidenti su monito delfico, imponendo la sua egemonia sulle popolazioni locali, per essere accettato come sovrano dopo avere unificato il territorio in modo coerente. ${ }^{42}$ Questa storia segna una pacificazione ispirata da Apollo, dunque sotto la più alta egida spirituale e sembra antitetica all'accusa contro Dionisio I di voler saccheggiare il santuario di Delfi, spogliandolo dei suoi tesori. ${ }^{43}$

Nel complesso, la tradizione tebana si combina con il motivo siciliano concorrente dell'ascendenza di Illirio da Polifemo, che rilancia in prospettiva culturale e politica l'espansionismo siracusano nei Balcani in via di ellenizzazione. Difatti, secondo tale mito gli Enchelei non sono stati sottomessi da dinasti di provenienza epicorica, ma sono sempre stati governati da sovrani di ascendenza greca. ${ }^{44}$ I mitici capostipiti sono identificati in alternativa a Polifemo-Galatea nella coppia Cadmo-Armonia, ove il sovrano di origine fenicia adombra una presenza semitica occidentale ed euboica ${ }^{45}$. Tali strategie di appropriazione etnica sono ancorate saldamente alla tradizione storico-mitica nazionale e rivelano elaborazioni sottese alle contingenze politiche. D'altra parte, i Siracusani rivitalizzano la leggenda diffusa nella costa dalmata, cioè nell'Illiria settentrionale, nelle colonie corinzie di Epidamno e Apollonia, ma la piegano alle esigenze ideologiche volte a suffragare la loro penetrazione nei Balcani occidentali. ${ }^{46}$

In ultima analisi, il ruolo del Ciclope etnogono non pare un'invenzione timaica. Lo storico di Tauromenio ha recepito un tema genealogico, la cui gestazione si situa in contesto eziologico-didascalico

${ }^{42}$ La leggenda di Cadmo e Armonia di paternità tebana è precocemente recepita nel patrimonio dei Corinzi e impiantata nell'Adriatico in accordo con l'espansione commerciale nell'età di Cipselo, cfr. Rossignoli 2004, 115.

${ }^{43}$ Cfr. Diod. 15.13.1. Sulla tradizione ascrivibile, ragionevolmente, all'opposizione contro il tiranno, cfr. Braccesi 1977, 190; Sordi 1999, 113; De Sensi Sestito 2011, 362.

${ }^{44}$ Gli Enchelei adriatici hanno lo stesso nome di una tribù tebana ( $>$ है $\gamma \chi \varepsilon \lambda \nu \varsigma$, "serpente d'acqua", vd. lat. anguilla) e richiamano la metamorfosi ofitica di Cadmo e Armonia, inoltre alludono a spostamenti fluviali o fluvio-terrestri, in corrispondenza della doppia localizzazione della tomba di Cadmo e Armonia in Istria a Pola e a Rhizon presso le Bocche di Cattaro vd. anche Ap. Rh. 4.516-518; Lycophr. Alex. 1026, con comm. Vian 1963, 126. Qui costituiscono un dominio potente alla metà del IV sec. a.C., cfr. ps.-Scy-

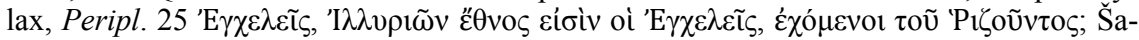
šel Kos 1993, 119-121; Rossignoli 2004, 108-109.

${ }^{45}$ Contatti col Vicino Oriente e mediazioni dell'Eubea sono presupposti da queste scaturigini mitiche, cfr. Šašel Kos 1993, 127, 130; Rossignoli 2004, 118.

${ }^{46}$ Cfr. Šašel Kos 1993, 123-124 per la popolarità della storia di Cadmo e Armonia in Illiria; Rossignoli 2004, 118: l'elemento epicorico dalmata e illirico è ricollegato a parentele greche sulla base di questa temperie ideologica. 
ad opera di un poeta di corte favorito dal mecenatismo di Dionisio il Grande, il quale coltiva personalmente ambizioni letterarie. ${ }^{47}$ Una creazione poetica dedicata agli amori di Polifemo e della nereide Galatea con sviluppi genealogici in chiave etnografica è assai plausibi-

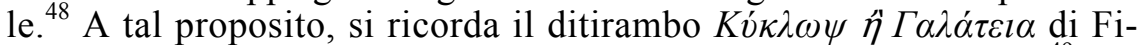
losseno di Citera (435-380 a.C.) iniziato alla corte siracusana, ${ }^{49} \mathrm{ma}$ eseguito ad Atene nel 390/389 e parodiato nella parodos del Pluto aristofaneo (388) in linea con l'indirizzo ateniese contrario alla spregiudicata politica adriatica di Dionisio il Vecchio. ${ }^{50}$ Filosseno veicola anche motivi di risentimento personale contro il tiranno e reca la più antica testimonianza sul gamos di Polifemo e Galatea, ${ }^{51}$ che è decisiva per l'evoluzione del personaggio del Ciclope innamorato recepita dalla Commedia di Mezzo ${ }^{52}$ e dal poeta siracusano Teocrito (315-260 a.C. ca.) in due Idilli da leggere in complementarità. In XI Galatea gioca il ruolo della ragazza altera e sdegnosa, la quale rifiuta la corte del suo rozzo corteggiatore monoculo, mentre in VI è Polifemo a respingere le profferte della Nereide innamorata. ${ }^{53}$

Parimenti è ragionevole il rilancio del motivo genealogico in discussione da parte di uno storico del partito dionigiano quale Filisto di Siracusa (430-356 a.C. ca.), ${ }^{54}$ responsabile della propaganda filo-

${ }^{47}$ Sono note le manie versificatorie del tiranno siracusano, autore di rapsodie fatte eseguire a Olimpia (Diod. 14.109.1-2) e tragedie rappresentate ad Atene sempre a sue spese (V. Eur. 5.14-18 Schw.; Cic. Tusc. 5.22), che suscitarono la parodia di Eubulo, Dionysius, fr. 25-26 K.-A., cfr Sanders 1987, 20-21; Castiglioni 2014, 28; Farmer 2017, 48-49. Per i rapporti tra il tiranno e gli intellettuali ateniesi, cfr. Bearzot 1981, 124-130; Intrieri 2015, 72 .

${ }^{48}$ Tale tesi formulata da Gruppe 1906, I, 391, n. 1 e accolta da Jacoby, comm. FGrH 566 F69, 375 n. 360, è rilanciata di recente da Briquel 1995, 244 e n. 31.

${ }^{49}=P M G$ 815-824, cfr. la testimonianza del peripatetico Phaenias, $f r .13$ Wehrly $=$ $P M G 816$ con interpretazione del ditirambo come satira politica, cfr. Gutzwiller 1991, 64; Hordern 1999, 445-446; Troiani 2018, 138.

${ }^{50}$ Cfr. Ar. Plu. 290-301 con Schol. ad 290 c-d 65 Chantry e 550, con un'allusione malevola: Penía, la Povertà, accusa Cremilo di non distinguere tra Dionisio e Trasibulo, cioè tra il tiranno e il liberatore, cfr. Alessandrì 1997, 140; Imperio 1998, 210; Hordern 1999; Muccioli 2004, 123; Gelli 2008, 245; Castiglioni 2014, 26-27, 31; Intrieri 2015, 72; Troiani 2018, 137, 140; Pierozzi 2019, 65.

${ }^{51}$ Cfr. Gutzwiller 1991, 64; Gelli 2008, 245; Castiglioni 2014, 26; Troiani 2018, 137.

${ }^{52} \mathrm{Nel}$ Ciclope di Antifane ( $f r$. 129-131 K.-A.), nella Galatea di Nicocare ( $f r$. 3-6 K.A.) e di Alessi ( $f r$. 37-40 K.-A.), in cui si accentua il processo di civilizzazione di Polifemo con effetti parodici, cfr. Gelli 2008, 246, 255-256; Castiglioni 2014, 26; Troiani 2018, 140.

${ }^{53}$ Cfr. Griffin 1983, 190; Gutzwilller 1991, 108-111, 124-126; Gutzko 2007, 101; Reed 2014; Lelli 2017, 267, 321. Per la tipologia dello «sprödes Mädchen», la ragazza sdegnosa, cfr. Kost 1971, 179; Costanza 2008, 127-137, in rapporto ai modelli teocritei di Galatea e Amarillide corteggiata, invano, dal capraio dell'Id. III, uno spasimante rozzo e sgraziato, un Doppelgänger di Polifemo (VI, XI).

${ }^{54}$ Sulla concezione politica della dynasteía di Dionisio I quale governo ideale in Filisto, cfr. Meister 2002, 459-461. 
barbarica orchestrata d'intesa con le direttive di Dionisio $I^{55}$ che è considerato un modello di azione politica da Alessandro Magno ${ }^{56}$. Seppure ascrivibile, come rilevato, all'opposizione antitirannica, Timeo non si esime dal riferire un motivo entrato nel repertorio della cultura etnografica impostata da Dionisio e diffuso poi nella cultura ellenistica. Un'eco della ricezione del tema si registra in Timagene di Alessandria, attivo come insegnante al tempo di Pompeo e di Augusto a Roma, dove giunse quale prigioniero di guerra. L'excursus gallico di Timagene riferito da Ammiano Marcellino testimonia la notizia di Celto, re eponimo, figlio di Galatea: $:^{57}$

Aborigenes primos in his regionibus quidam uisos esse firmarunt; Celtos nomine regis amabilis et matris eius uocabulo $\mathrm{Ga}$ latas dictos - ita enim Gallos sermo Graecus appellat.

Alcuni asseriscono che i primi abitanti attestati in queste regioni sono indigeni, questi furono chiamati Celti dal nome di un re amato e Galati dall'appellativo della madre di quest'ultimo, pertanto, in lingua greca sono denominati Galli.

Fin dall'età ellenistica, il Ciclope è connesso stabilmente alla tradizione della Sicilia greca, i suoi amori con Galatea sono cantati alla corte dionigiana e la sua figura permane un elemento chiave per i dinasti siracusani. Secondo la testimonianza di Plutarco, gli Itacesi accusano Agatocle (360 ca. - 289 a.C.) dei saccheggi contro di loro e contro i Corciresi, ma l'autoproclamato basileus siracusano replica in tono sprezzante che il loro re Odisseo era giunto in Sicilia e aveva accecato il pastore Polifemo, pertanto, legittima le sue azioni di disturbo nelle isole ioniche come la vendetta del torto ancestrale contro il $\mathrm{Ci}$ clope. $^{58}$

${ }^{55}$ Cfr. Briquel 1995, 245 sulla scorta di una proposta avanzata da Braccesi 1991a, 9192; Vanotti 1993, 119-120; Sordi 1999. Sulla linea ideologica dionigiana favorevole a una syngéneia europea attivata da Filisto, cfr. Sammartano 2008, 138-146; Troiani 2018, 153 ; Pierozzi 2019, 64. La matrice siracusana permane la prospettiva cogente per la leggenda genealogica che non è nata ad Atene, come ipotizza Castiglioni 2014, 31: le detorsioni comiche sfruttano il tema, amplificando l'accusa contro l'attitudine filobarbarica della grecità d'Occidente.

${ }^{56}$ Cfr. Sordi 1985; Berlinzani 2008, 120-121: fra i libri che il sovrano macedone fa pervenire in Asia tramite Arpalo si enumerano gli scritti storici di Filisto e i ditirambi di Filosseno (Plu. Al. 8.3-4).

${ }^{57}$ 15.3.9 (FGrH 588 F69), Celto e Galato paiono qui fusi in un'unica personalità, mentre la mater citata non può che essere la Nereide amata dal Ciclope, cfr. l'analisi di Briquel 1995, 241-243; in genere Sordi 1982, 778-779; Muccioli 2001, 356, 364: la tradizione di Timagene è indipendente dal Libro illirico di Appiano e riporta pure versioni alternative.

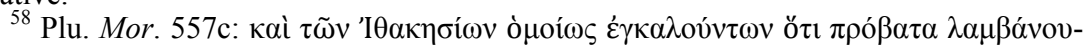

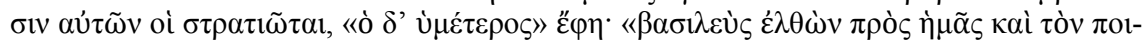
$\mu \varepsilon \dot{v} \alpha \pi \rho \sigma \sigma \varepsilon \xi \varepsilon \tau \dot{\varphi} \varphi \lambda \omega \sigma \varepsilon v$, cfr. De Vido 2015,185 con ipotesi che «tutti i lembi di racconto di argomento più o meno latamente 'odissiaco', pur frammentari, possano rimandare a un ambiente culturalmente omogeneo di matrice siracusana, abbastanza autonomo e spregiudicato da impugnare le più antiche tradizioni greche». 


\section{Fonti umanistiche}

È meritevole d'interesse il riuso umanistico della storia di Polifemo e Galatea ancora con finalità eminentemente politiche. Alla fine del XV secolo Francesco Filelfo (Tolentino 1398 - Firenze 1481) adduce il motivo dei tre figli del Ciclope quali progenitori dei rispettivi popoli in vista dell'organizzazione di un'auspicata campagna anti-ottomana. In una lettera al doge di Venezia Nicolò Tron del 1471 ripropone il mito della discendenza ciclopica, in cui inserisce le origini del popolo magiaro attraverso l'anello intermedio del sovrano eponimo Ungaro. ${ }^{59}$ Nella rielaborazione di Filelfo è palese la dipendenza dall'erudizione classica, in special modo da Appiano utilizzato senza essere citato. Lo storico alessandrino rientra fra gli auctores prediletti dall'umanista, il quale ne curò un latinizzamento perduto. ${ }^{60}$

Il tema genealogico è riesumato per esaltare il furor bellico delle forze ungheresi guidate da Mattia Corvino (Hunyadi Mátiás, 14581490) indicato come l'alleato ideale di Venezia nella lotta al Turco, in cui l'anziano intellettuale rivendica instancabilmente per sé il ruolo di consigliere quale alter Nestor. ${ }^{61}$ Nello schema classico relativo all'etnogenesi europea Filelfo inserisce opportunamente i Magiari, senza dimenticare gli Unni e il loro re Attila. Il Tolentinate è un erede fedele della notizia elaborata alla Corte dionigiana, non ripete stancamente una curiosità mitologica, ma sfrutta quest'artificio per evidenziare la parentela dell'Ungheria con i popoli dell'Europa occidentale, specialmente Italiani e Francesi quali discendenti diretti dei Celti. La fratellanza dei Magiari con tali popoli diventa d'importanza cruciale in vista di un'alleanza stabile tra il Regno di S. Stefano, la Serenissima e la Corona di Francia per organizzare un fronte comune contro i nemici della Cristianità. Giova riportare l'excursus filelfiano: ${ }^{62}$

[...] Ex omni Christianorum genere Pannones et qui Pannoniam superiorem Hungari incolunt et aptissimi sunt et acerrimi, qui congrediantur cum Turcis in pugnam. Nam et finitimi sunt et quotidianis fere praeliis adversus Turcos exercitati. Atque, ut intelligatur, qui sint Pannones et qui Hungari, paulo altius repetam et id quidem paucis. Polyphemo cognomento Cyclopi ex Galathea filii tres nati perhibentur Celtus, Illyricus, Gallas, quicum ex Sicilia movissent, tribus his nationibus bello superatis imperitarunt Celtis, Illyricis atque Gallis, quibus etiam singuli a suis nominibus nomina indiderunt. Sed ex Illyrici posteritate nati

\footnotetext{
${ }^{59}$ Cfr. Costanza 2020, 321.

${ }^{60}$ Filelfo approntò febbrilmente una traduzione di Appiano in aperta concorrenza col lavoro già realizzato dal suo rivale alla Corte milanese Pier Candido Decembrio, cfr. Calderini 1913, 258-60; Bianca 1986, 233; Zaggio-Mulas-Ceriana 1997, 115-117.

${ }^{61}$ Cfr. Costanza 2015, 37-44; Id. 2019, 122-128.

${ }^{62}$ Ep. XXXIV 23, da Milano, 4/12/1471, ed. de Keyser 2017, III 1473-1479, spec. 1476, 125-1477, 169; Costanza 2020, 336-337.
} 
autumantur Pannonius aut Pannon et Scordiscus et Paeon ac Triballus, qui etiam a suis ipsorum nominibus gentis, quas subiugarunt, appellavere. Pannones igitur iisdemque Pannonii ex Polyphemo illo ducentes originem, qui belli Troiani temporibus in Sicilia regnans vir fuit bellicosissimus et arcus sagiptaeque peritissimus, tam ex paterno sanguine quam ex assidua exercitatione nihil magis oderunt quam otium ac pacem. Quare bellum et pugnam habent pro animorum relaxatione ac ludo. Quid autem Hungari appellentur, id nomen ab incolis Scythis accepere. Nam Scythici quidam populi, quibus Hunnis nomen fuit, relicto patrio regno, in superiore Pannonia consedere, qui postea et rege et duce Atila in Italiam manu praedatoria descenderunt, Leone viro illo sanctissimo, urbis Romae Pontifice Maximo. Is, audita irruptione hostis tam formidabilis, tam horrendi, tam truculenti, nullo retardatus metu, quin fraetus divina ope bonaeque spei plenus Atilae ipsi in ordine et ornatu pontificali occurrit ad Padum, cum iam esset in transitu. Quod Atilas cum audisset, non substitit modo, sed advenientem pontificem reverenter excepit eumque dicentem perhumaniter audivit et quod petierat, perbenigne concessit. Petierat autem aliud nihil praeter id unum, ne Padum traiiceret. Consyderaverat enim pontifex sapientissimus sibi atque Italiae satis fore, si ab homine saevicia efferato atque praedae avido extorqueret, ne interius Christianos populos discerperet. Iam enim post incredibilem hominum omne genus caedem, Aquileiam urbem nobilem ac vetustam solo aequarat et universam fere Venetiam diripuerat ac vastarat. Ex illo aut naufragio tam tetro, tam luctuoso, tam horribili quicunque viri nobiles et potentes evadere potuerunt, ii undique in tutissimum locum istum convenientes, urbem condidere Venetias, quam vos viri patricii ex illo tempore in hanc diem quanfortunatissime pacatissimeque incolitis sine ulla tanti imperii mutabilitate, id quid neque Atheniensibus, neque Lacedaemoniis, neque Carthaginiensibus, nec Romanis ipsis, nec ulli unquam aut uniuscuiusquam imperio aut reipublicae contigit. Et, ut id vobis perpetuum sit, immortalem Deum oro atque obsecro. Nam et Atilas post Mediolanum in Insubribus, quo diverterat, radicitus excisum, in Pannoniam revertit et qui deinceps Gotthi primo, dein Longobardi ex Hungaria, nam Pannonia hoc postea nomina ab Hungaro est rege appellata et alii quotquot Italiam saepe numero invasere, nihil omnino vobis incommodarunt, quod eius rei multo magis vestrae et concordiae et prudentiae atque iusticiae dandum reor quam loco ulli vel natura vel arte hominum munitissimo. $[\ldots]$

[...] Fra tutti i Cristiani i Pannoni e gli Ungheresi, i quali abitano la Pannonia superiore [cioè l'Ungheria], sono $i$ più idonei alla lotta e i più forti per la guerra contro il Turco. Sono i confinanti dei Turchi e sono addestrati dai combattimenti quasi quoti- 
diani contro di loro. Per far capire, chi siano i Pannoni e gli Ungheresi, ripeterò una notizia antica in poche parole: si dice che Galatea e Polifemo generarono tre figli di nome Celto, Illirico e Galla. Costoro partirono dalla Sicilia, sconfissero in battaglia tre nazioni e sottomisero i Celti, Illiri e Galati, i quali presero nome da loro. Si racconta riguardo alla discendenza di Illirico, che da lui nacquero Pannonio (o Pannone), Scordisco, Peone e Triballo. $\mathrm{Da}$ essi presero nome i popoli che essi avevano in precedenza sgominato. I Pannoni dunque trassero origine da Pannonio e dal celebre Polifemo, il quale regnava all'epoca della guerra troiana in Sicilia ed era un uomo molto bellicoso e un maestro con l'arco e frecce. Di conseguenza, gli Ungheresi, in ragione del sangue paterno e del loro stabile esercizio, non odiano nulla più dell'ignavia e della pace e reputano la guerra e la battaglia uno svago dell'animo e un gioco. Perché gli Ungheresi siano stati chiamati così, trassero il loro nome dagli abitanti scitici. Quindi, s'insediarono alcuni popoli scitici, che furono chiamati Unni, nella Pannonia superiore, dopo aver lasciato il loro paese. In seguito, si diressero con il loro re Attila in Italia, quando il Sommo Pontefice a Roma era Papa Leone. Dopo aver udito di un attacco di un nemico così cruento, orrendo, terribile, mosse verso Attila fidando nell'aiuto divino e pieno di speranza, accorre al Po senza indugio e con le vesti pontificali, quando l'altro era già in viaggio. Quando Attila lo apprese, non solo si fermò, ma lo accolse con riverenza, lo ascoltò parlare con umanità e concesse con grande benevolenza ciò che chiedeva. Quegli non chiedeva nient'altro se non di non attraversare il Po. Il Papa assai sapiente aveva considerato che sarebbe stato sufficiente per sé e per l'Italia, stornare quell'uomo efferato e avido di bottino, affinché non vessasse ulteriormente i popoli cristiani. Infatti, dopo un'inaudita strage, aveva raso al suolo la nobile e antica città di Aquileia e distrutto e devastato quasi tutto il Veneto. Da quel naufragio così triste, ferale e orrendo tutti gli uomini nobili e potenti che riuscirono a scampare, radunandosi da ogni parte in questo luogo assai sicuro e fondarono la città di Venezia, che voi, patrizi, abitate da quel tempo fino a oggi in condizioni fauste e pacifiche senz'alcun cambiamento di un potere così stabile, che non è toccato ad Ateniesi, Spartani, Cartaginesi e neppure ai Romani come a nessun altro regno o repubblica. E prego e scongiuro Dio che così perduri sempre. Infatti, Attila, dopo avere distrutto alla radice Milano in Lombardia, dove si era diretto, ritornò in Pannonia: i Goti prima, i Longobardi poi e altri i quali invasero spesso in gran numero l'Italia dall'Ungheria, cioè la Pannonia così ridenominata dal re Ungaro, non vi hanno recato alcun fastidio; ritengo che ciò sia dovuto molto di più alla vostra concordia, prudenza e giustizia più che alla posizione del luogo fortificato dalla natura e dalla tecnica umana. [...] 
Nell'ambito degli interessi eruditi rinascimentali si segnala la notizia antiquaria elaborata da Natale Conti, alias Natalis Comes (Milano 1520-1581) nella sua enciclopedia mitografica, in cui ogni mito è spiegato in chiave moralistica a fini didattici ${ }^{63}$. In quest'opera variamente ristampata tra la fine del XVI e del XVII secolo la prole di Polifemo e Galatea è, ulteriormente, ampliata rispetto alla triade nota dalla tradizione antica con l'aggiunta di altri due fratelli, Eneto e Paflagono, ${ }^{64} \mathrm{i}$ quali sono investiti del ruolo di eponimi pertinente ad ambiti geografici che non erano estranei agli interessi di penetrazione della Siracusa dei due Dionigi: ${ }^{65}$

Dicitur Polyphemus non modo amasse Galateam, sed etiam Galatum ex ea suscepisse, ut testatus est Bacchylides (Fr. 59 Sn.M.), cum quidam etiam Polyphemi filium fuisse inquiant Celtum, a quo dicti sunt Celtae, et Illyrium, a quo Illyri, et Henetum, ut quidem voluerunt, a quo regio postea Venetia, et Paphlagonum, ut ait Dercyllus in libro de nominibus urbium et locorum.

Si narra che Polifemo non solo abbia amato Galatea, ma abbia anche concepito con lei Galato, come attesta Bacchilide, mentre alcuni asseriscono che era figlio di Polifemo anche Celto e Illirio, dal quale presero nome i Celti e gli Illiri e, come pretesero alcuni, Eneto, dal quale fu chiamata la regione Veneto e Paflagono, come dice Dercillo nel libro Sui nomi delle città e dei luoghi.

Nonostante il credito accordato a vario titolo a questa testimonianza, ${ }^{66}$ tale citazione isolata non consente di retrodatare la genealogia di Polifemo e Galatea al poeta lirico del V secolo a.C. È preferibile ancorarne la genesi all'età dionigiana in accordo con le fonti sopra discusse e il contesto storico-politico di riferimento. ${ }^{67}$

${ }^{63}$ Vd. notizia biobibliografica in Ricciardi 1983.

${ }^{64}$ Natalis Comes, Mythologiae sive explicationis fabularum libri decem. In quibus omnia prope naturalis et moralis philosophiae dogmata sub antiquorum fabulis contenta fuisse demonstratur (1561-64) 1. 9, cap. 8 De Cyclopibus, l'etnogonia in questa forma ampliata e il rinvio a Bacchilide restano incontrollati, cfr. Vattuone 1991, 134 e n. 38; Briquel 1995, 243 e n. 25; Alessandrì 1997, 140. Sul legame tra Veneti e Paflagoni, cfr. Šašel Kos 1993, 113 sulla scorta di Polacco 1985.

${ }^{65}$ Sull'interesse dionigiano per i Veneti italici in quanto coloni dei Celti, cfr. Coppola 2000, 13, 17-18 per leggende troiane e colonie enetiche in Paflagonia, regione anatolica tra Bitinia e Cappadocia.

${ }^{66}$ Cfr. Coppola 2000, 13-14; Ead. 2002, 380; Muccioli 2004, 129; Troiani 2018, 142.

${ }^{67}$ Cfr. Anello 1984, 22; Troiani 2018, 142-143; Pierozzi 2019, 62. N. Comes può essere reputato il compilatore di un'abile falsificazione, come ammette Coppola 2002, 380 . 


\section{Conclusioni}

In definitiva, la scelta di eroi eponimi ellenici nel IV secolo a.C. è dettata dalla necessità politica di stabilire una syngéneia con Siracusa, al fine di legittimare storicamente la pretesa della dynasteía sull' Adriatico vagheggiata da Dionisio il Vecchio. I riferimenti etnografici evocati nella genealogia in esame riflettono gli interessi dirimenti dell'espansione dionigiana e le sue direttrici precipue. Questa costruzione ideologica favorisce una penetrazione culturale ad ampio raggio e postula condizioni d'incontro propizie con il mondo illirico e celtico col risultato di eroderne la dimensione di alterità a vantaggio di un'interazione su solide basi comuni. L'etnogonia postulata dalle fonti convalida il progetto ambizioso di una talassocrazia, in cui compagini differenti condividono orizzonti politici e latamente culturali, cementando l'egemonia siracusana. La struttura mitologica imbastita in un ambiente vicino al tiranno testimonia l'aspirazione all'assimilazione alla grecità dei bellicosi popoli dell'Occidente e dell'Illirico elevati ad uno statuto di assoluto prestigio. La progenie mitica convalida l'ascendenza divina di Celti e Illiri da Posidonio e Nereo, il Vegliardo del mare, in quanto padri del Ciclope e della Nereide. La solenne archaiologia configura vincoli di parentela con funzione nobilitante tra genti diverse e le divinità marine, nella prospettiva di un Mediterraneo unificato come un Mare nostrum siracusano.

\section{BIBLIOGRAFIA}

Alessandrì, Salvatore. “Alessandro Magno e i Celti”. Museum Helveticum 54.3, 1997, 131-157.

Alfieri Tonini, Teresa - Struffolino, Stefano (edd.). Dinamiche culturali ed etniche nella Sicilia orientale (Aristonothos. Scritti per il Mediterraneo antico, Quaderni 4). Trento, Tangram, 2014.

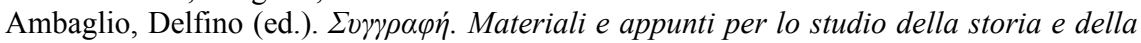
letteratura antica 6. Como, New Press, 2004.

Anello, Pietrina. Dionisio il Vecchio I. Politica adriatica e tirrenica. Palermo, Boccone del Povero, 1980.

Anello, Pietrina. "Polifemo e Galatea". Seia 1, 1984, 9-51.

Anello, Pietrina. "Note sui rapporti tra Dionisio I e Atene nel primo decennio del IV secolo". Kokalos 42, 1996, 383-408.

Anello, Pietrina. "La colonizzazione siracusana in Adriatico". In Braccesi-Graciotti (edd.), 1999, 117-146.

Anello, Pietrina - Martínez Pinna, Jorge (edd.). Relaciones interculturales en el Mediterráneo antiguo: Sicilia e Iberia = Rapporti interculturali nel Mediterraneo antico. Málaga, Servicio de Publicaciones, Centro de Ediciones de la Diputación - Palermo, Università degli Studi, 2008. 
Antonetti, Claudia. "Lanassa e Corcira: la sposa, l'isola, la culturalità demetriaca al servizio della regalità ellenistica". In Ead.-Cavalli (edd.), 2015, 191-219.

Antonetti, Claudia - Cavalli, Edoardo (edd.). Prospettive corciresi (Diabaseis 5). Pisa, ETS, 2015.

Avesani, Rino et al. (edd.). Francesco Filelfo nel quinto centenario della morte. Atti del XVII Convegno di Studi Maceratesi (Tolentino, 27-30 settembre 1981), (Medioevo e Umanesimo 58; Studi Maceratesi 17). Padova, Antenore, 1986.

Balard, Michel (ed.). Eúuvxía. Mélanges offerts à Hélène Ahrweiler, I-II, Paris, Publications de la Sorbonne, 1998.

Bearzot, Cinzia. Platone e $i$ "moderati" ateniesi. Milano, Istituto lombardo di scienze e lettere, 1981.

Bearzot, Cinzia. "I Celti in Illiria. A proposito del frg. 40 di Teopompo". In Urso (ed.), 2004, 63-78.

Bearzot, Cinzia - Vattuone, Riccardo - Ambaglio, Delfino (edd.). Storiografia locale e storiografia universale. Forme di acquisizione del sapere storico nella cultura antica. Atti del Congresso Bologna, 16-18 dicembre 1999. Como, New Press, 2001.

Belardinelli, Anna Maria - Imperio, Olimpia - Mastromarco, Giuseppe - Pellegrino, Matteo - Totaro, Piero (edd.). Tessere: frammenti della commedia greca, studi e commenti. Bari, Adriatica, 1998.

Berlinzani, Francesca. "Teleste di Selinunte il ditirambografo". Aristonothos 2, 2008, 109-140.

Bertrand, Dominique. (ed.). Mythologies de l'Etna. (Collections volcaniques). ClermontFerrand, Presses Universitaires Blaise Pascal, 2004.

Bianca, Concetta. "Auctoritas e veritas: il Filelfo e le dispute tra platonici e aristotelici". In Avesani (ed.), 1986, 207-247.

Bonacasa, Nicola - Braccesi, Lorenzo - De Miro, Ernesto (edd.). La Sicilia dei due Dionisî. Atti della settimana di studio Agrigento, 24-28 febbraio 1999, Akragas 2, Roma, L'Erma di Bretschneider, 2002.

Braccesi, Lorenzo. Grecità adriatica. Un capitolo della colonizzazione greca in Occidente. Bologna, Pàtron, $1977^{2}$.

Braccesi, Lorenzo. "Diomedes cum Gallis.” Hesperia 2, 1991a, 89-102.

Braccesi, Lorenzo. "Ancora sulla colonizzazione siracusana in Adriatico (Dionigi, Diomede e i Galli)." In Cassio-Musti (edd.), 1991b, 57-64.

Braccesi, Lorenzo. Grecità di frontiera. I percorsi occidentali della leggenda. Padova, Esedra, 1994.

Braccesi, Lorenzo (ed.). La pirateria nell'Adriatico antico. Atti dell'Incontro di Studio Venezia, 10 marzo 2002 = Hesperia 19, Roma, L'Erma di Bretschneider, 2004.

Braccesi, Lorenzo - Graciotti, Sante (edd.). La Dalmazia e l'altra sponda. Problemi di archaiologhia adriatica (Venezia, 16-17 gennaio 1996). Fondazione Giorgio Cini Civiltà Veneziana, Studi 50, Firenze, Olschki, 1999.

Braccesi, Lorenzo - Luni, Mario (edd.). Greci in Adriatico. Atti del Convegno internazionale $($ Urbino, 21-24 ottobre 1999) = Hesperìa 15, Roma, L'Erma di Bretschneider, 2002.

Briquel, Dominique. "Des Aborigènes en Gaule: à propos d'un fragment de Timagène". Hesperìa 5, Roma, L'Erma di Bretschneider, 1995, 239-249.

Broilo, Fulviomario (ed.). Xenia. Scritti in onore di Pietro Treves (La Fenice 3). Roma, L'Erma di Bretschneider, 1985.

Burzacchini, Gabriele. "Eur. Cycl. 320-331". Quaderni Urbinati di Cultura Classica 32, 1979, 65-68.

Cabanes, Pierre. “Greek Colonization in Hadriatic”. In Tsetskhladze (ed.), 2008, 155-186.

Caccamo Caltabiano, Maria. "La monetazione di Dionisio I fra economia e propaganda". In Bonacasa - Braccesi - De Miro (edd.), 2002, 33-46.

Calderini, Aristide. "Ricerche intorno alla biblioteca e alla cultura greca di Francesco Filelfo". Studi italiani di Filologia Classica 20, 1913, 204-424. 
Cassio, Albio Cesare - Musti, Domenico (edd.). Tra Sicilia e Magna Grecia. Aspetti di interazione culturale nel IV sec. a.C. Atti del Convegno Napoli 19-20 marzo 1987 = AION (filol.) 11. Roma, Ateneo, 1991.

Castiglioni, Maria Paola. "La discendenza di Polifemo e la politica di Dionigi di Siracusa". In Alfieri Tonini-Struffolino (edd.), 2014, 25-34.

Caven, Brian. Dionysius I: War-Lord of Syracuse. New Haven-London, Yale University, 1990.

Cioppa, Margherita. "La preistoria della Dalmazia - Cadmo e Armonia". Rivista Dalmatica 54.3, 1993, 205-213.

Class, James Joseph - Cuypers, Martine (edd.). A Companion to Hellenistic Literature. Chichester, Wiley-Blackwell, 2014.

Consolo Langher, Sebastiana N. Un imperialismo tra democrazia e tirannide. Siracusa nei secoli V e IV a.C. Roma, L'Erma di Bretschneider, 1997.

Consolo Langher, Sebastiana N. "Corcira e l'Adriatico negli equilibri interstatali del Mediterraneo tra IV e III sec. (età di Agatocle e dei Diadochi)". In Braccese-Luni (edd.), 2002, 73-81.

Coppola, Alessandra. "Siracusa e il Diomede adriatico". Prom. 14, 1988, 221-226.

Coppola, Alessandra. "Ancora su Celti, Iperborei e propaganda dionigiana". Hesperia 2, Roma, L'Erma di Bretschneider, 1991, 103-106.

Coppola, Alessandra. Demetrio di Faro. Un protagonista dimenticato (Problemi e ricerche di storia antica 15). Roma, L'Erma di Bretschneider, 1993a.

Coppola, Alessandra. "L'occidente: mire ateniesi e trame propagandistiche siracusane". Hesperia 3, Roma, L'Erma di Bretschneider, 1993b, 99-113.

Coppola, Alessandra. Archaiología e propaganda. I Greci, Roma e l'Italia (Problemi e ricerche di storia antica 18), Roma, L'Erma di Bretschneider, 1995.

Coppola, Alessandra. "La leggenda troiana in area venetica". Hesperì 12, Roma, L'Erma di Bretschneider, 2000, 11-22.

Coppola, Alessandra. "Mito e propaganda alla corte dionisiana". In Bonacasa-BraccesiDe Miro (edd.), 2002, 372-388.

Costanza, Salvatore. "Teocrito Id. III: l'indovino Melampo ed il manifesto palmomantico" Eikasmos 19, 2008, 127-150.

Costanza, Salvatore. La divinazione greco-romana. Dizionario delle mantiche: metodi, testi e protagonisti, Udine, Forum, 2009.

Costanza, Salvatore. 'Testimonianze epistolari sulla caduta dell'Eubea (1470): La posizione di Filelfo, alter Nestor". In Fiaschi (ed.), 2015, 25-46.

Costanza, Salvatore. "Die Reise des Johannes VIII. Palaiologos laut Francesco Filelfo und anderer Quelle". In Juhász (ed.), 2019, 61-84.

Costanza, Salvatore. "Ungarn, Byzantinische Welt und Türkenkrieg in Filelfos Episteln". In Juhász (ed.), 2020, 311-346.

Cusset, Christophe. "L'Etna dans le Cyclope d'Euripide". In Bertrand (ed.), 2004, 25-32.

D'Andria, Francesco. "L'Adriatico. I rapporti tra le due sponde: stato della questione". In Bonacasa-Braccesi-De Miro (edd.), 2002, 117-137.

de Keyser, Jeroen (ed.), Francesco Filelfo. Collected Letters. Epistolarum libri XLVIII. IIV. Alessandria, dell'Orso, 2017.

Demandt, Alexander. Die Kelten. München, C.H. Beck, 1998.

De Sensi Sestito, Giovanna. "Magna Grecia, Epiro e Sicilia fra IV e III sec. a.C.: spinte egemoniche a confronto". In Ead.-Intrieri (edd.), 2011, 361-390.

De Sensi Sestito, Giovanna - Intrieri, Maria (edd.). Sulla rotta per la Sicilia: L'Epiro, Corcira e l'Occidente. Pisa, ETS, 2011.

De Vido, Stefania. "Tyrannos, strategos autokrator, dynastes. Le ambigue parole del potere nella Sicilia di IV secolo". In Mari-Thornton (edd.), 2013, 45-60.

De Vido, Stefania. "Il re Agatocle nello spazio ionico: prospettive e modelli". In AntonettiCavalli (edd.), 2015, 169-190.

Dzino, Danijel. Illyricum in Roman Politics 229 BC-AD 68, Cambridge, University, 2010. 
Ercolani, Andrea - Livadiotti, Umberto (trad./comm.). Appiano. La conquista romana dei Balcani: libro illirico, Lecce, Argo, 2009.

Farmer, Matthew C. Tragedy on the Comic Stage. Oxford, University, 2017.

Ferone, Claudio. "Appiano, Illyr. 3 e la pirateria illirica nel IV sec. a.C.". Herm. 132.3, 2004a, 326-337.

Ferone, Claudio. "Il IV secolo, Atene e l'Adriatico". In Braccesi (ed.), 2004b, 31-48.

Fiaschi, Silvia (ed.). Philelphiana. Nuove prospettive di ricerca sulla figura di Francesco Filelfo. Atti del seminario di studi. Macerata, 6-7 novembre 2013 (Quaderni di Rinascimento 51), Firenze, Olschki, 2015.

Gabba, Emilio. "Appiano traduttore in B.C. V 191". In Studi di storiografia antica in memoria di Leonardo Ferrero, Torino, Bottega d'Erasmo, 1971, 185-189.

Gabba, Emilio - Vallet, Georges (edd.). Storia della Sicilia. Napoli, Società editrice storia di Napoli e della Sicilia, 1979.

Gelli, Emiliano. "Euripide, Filosseno e il Ciclope di Antifane". Prometheus 34, 2008, 245-256.

Goukowsky, Paul. Appien, Histoire romaine, tome V, Livre IX, le livre Illyrien. Fragments du livre macédonien. Paris, Les Belles Lettres, 2011.

Griffin, Alan H.F. "Unrequited Love: Polyphemus and Galatea in Ovid's Metamorphoses". Greece \& Rome 30.2, 1983, 190-197.

Gruppe, Otto. Griechische Mythologie und Religionsgeschichte. München, C.H. Beck, 1906.

Gutzwiller, Kathryn J. Theocritus' Pastoral Analogies. The Formation of a Genre. Madison/WI, University of Wisconsin, 1991.

Hahn, István. “Appian und seine Quellen”. In Wirth (ed.), 1982, 251-276.

Hordern, James H. "The Cyclops of Philoxenus". Classical Quarterly 49.2, 1999, 445455.

Imperio, Olimpia. "Callias". In Belardinelli-Ead.-Mastromarco-Pellegrino-Totaro, edd., 1998, 195-254.

Intrieri, Maria. "Atene, Corcira e le isole dello Ionio (415-344 a.C.)". In Antonetti-Cavalli (edd.), 2015, 53-118.

Juhász, Erika (ed.). Byzanz und das Abendland VI. Studia Byzantino-Occidentalia. Budapest, Eötvös-József-Collegium, 2019.

Juhász, Erika (ed.). Byzanz und das Abendland VII. Studia Byzantino-Occidentalia. Budapest, Eötvös-József-Collegium, 2020.

Kost, Karlheinz (ed.). Musaios, Hero und Leander, Bonn, Bouvier, 1971.

Krutas, Venceslav. Les Celtes. Histoire et Dictionnaire. Paris, Chêne, 2000.

Kutzko, David. "The Bemused Singer and Well-versed Audience: The Use of Polyphemus in Theocritus". Studi Italiani di Filologia Classica 100, 2007, 73-114.

Lelli, Emanuele. Pastori antichi e moderni. Teocrito e le origini popolari della poesia bucolica (Spudasmata 174). Hildesheim-Zürich-New York, G. Olms, 2017.

Livadiotti, Umberto. Rec. Goukowsky 2011, Annali Online di Ferrara - Lettere 7.2, 2012, 176-182.

Lombardo, Mario. "La colonizzazione adriatica in età dionigiana". In Bonacasa-BraccesiDe Miro (edd.), 2002, 427-442.

Lombardo, Mario. "Il Canale d'Otranto tra il IV e il III secolo". In Braccesi (ed.), 2004, 49-59.

Maier, Bernhard. Geschichte und Kultur der Kelten. München, C.H. Beck, 2012.

Malamut, Élisabeth. "Concepts et réalités : recherches sur les termes désignant les serbes et les pays serbes dans les sources byzantines des X ${ }^{\mathrm{e}}-\mathrm{XII}{ }^{\mathrm{e}}$ siècles”. In Balard (ed.), 1998, 439-457.

Marasco, Gabriele. "L'«Illyriké» di Appiano". Aufstieg und Niedergang der Römischen Welt 2.34.1, Berlin, 1993, 463-495. 
Mari, Manuela - Thornton, John (edd.). Parole in movimento. Linguaggio politico e lessico storiografico, nel mondo ellenistico. Atti del Convegno Internazionale (Roma, 21-23 febbraio 2011), (Studi Ellenistici 27). Pisa-Roma, F. Serra, 2013.

Mastromarco, Giuseppe. "La degradazione del mostro. La maschera del Ciclope nella commedia e nel dramma satiresco del quinto secolo a.C.". In Belardinelli-Imperio-Id.-Pellegrino-Totaro (edd.), 1998, 9-42.

Mastrocinque, Attilio. Da Cnido a Corcira Melaina: uno studio sulle fondazioni greche in Adriatico (Pubbl. Dip. Storia della Civiltà Europea 4). Trento, Università degli studi, 1988.

Meister, Klaus. "Filisto e la tirannide". In Bonacasa-Braccesi-De Miro (edd.), 2002, 453462.

Merante, Vitaliano. "La Sicilia e Cartagine dal V secolo alla conquista romana". Kokalos 18-19, 1972-73, 77-107.

Moscati Castelnuovo, Luisa. "I Galeoti e Dodona: Filisto o Prosseno". In Struffolino (ed.), 2014, 17-24.

Muccioli, Federicomaria. "Interessi etnografici e tradizioni storiografiche nel Libro celtico di Appiano". In Bearzot-Vattuone-Ambaglio (edd.), 2001, 347-377.

Muccioli, Federicomaria. "Filosseno di Citera, Dionisio I e la fortuna del mito di Polifemo e Galatea tra IV e III secolo a.C.". In Ambaglio (ed.), 2004, 121-147.

Occhipinti, Egidia. "Dionisio il Vecchio e il consenso delle popolazioni anelleniche della Sicilia". Hormos 8, 2006, 65-82.

Pierozzi, Andrea. "Dionisio I, i Celti e il sacco di Roma. Alcune riflessioni sulla cronologia e sulla strategia delle operazioni militari siracusane tra l'Elleporo e Pyrgi". Erga - Logoi 7, 2019, 45-82.

Polacco, Luigi. “"Aponus” in Paflagonia". Quaderni ticinesi di Numismatica e Antichità classiche 14, 1985, 15-30.

Pontani, Anna (ed.), Niceta Coniata. Grandezza e catastrofe di Bisanzio (Narrazione cronologica). I Milano, Mondadori, 2017.

Reed, Joseph D. "Idyll 6 and the Development of the Bucolic after Theocritus". In ClaussCuypers (edd.), 2014, 238-250.

Rendić-Miočević, Duje. "I Greci in Dalmazia e i loro rapporti con il mondo illirico". In Atti Convegno di Cortona (24-30 maggio 1981) Forme di contatto e processi di trasformazione nelle società antiche = Publications de l'École française de Rome 67, 1983, 187-202.

Ricciardi, Roberto. "Conti (Comes, Comitum, De comitibus), Natale (Hieronymus)". In Dizionario Biografico degli Italiani 28, 1983, 454-457.

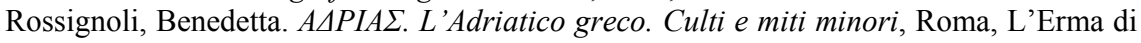
Bretschneider, 2004.

Sammartano, Roberto. "Filisto e l'origine delle popolazioni panelleniche di Sicilia". In Anello-Martínez Pinna (edd.), 2008, 115-146.

Sanders, Lionel Jehuda. Dionysius I of Syracuse and Greek Tyranny. London, Croom Helm, 1987, rist. London-New York, Routledge, 2014.

Santamato, Emanuele. "Il discorso di Bruto (BC 2.19.138-140) e l'introduzione ai Bella Civilia (BC 1.1.7-8) in Appiano: studio sulla comunicazione appianea”. Göttinger Forum für Altertumswissenschaft 16, 2013, 237-277.

Šašel Kos, Marjeta. "Cadmus and Harmonia in Illyria”. Arheološki vestnik 44, 1993, 113 136.

Šašel Kos, Marjeta. "The Roman Conquest of Dalmatia in the Light of Appian's Illyrike". In Urso (ed.), 2004, 141-166.

Šašel Kos, Marjeta. Appian and Illyricum (Situla. Razprave Narodnega muzeja Slovenije 43). Ljubljana, Narodni muzej Slovenije, 2005.

Šašel Kos, Marjeta. "Illyria and Illyrians”. In Bagnall (ed.), 3407-09.

Scarpa Bonazza Buora, Alessandra. Libertà e tirannide in un discorso "siracusano» di Diodoro Siculo, Roma, L'Erma di Bretschneider, 1984. 
Scarpi, Paolo (cur.) - Ciani, Maria Grazia (trad.). Apollodoro. I miti greci, Milano, Mondadori, 1996.

Shaw, Carl A. Satyric Play. The Evolution of Greek Comedy and Satyr Drama. Oxford, University, 2104.

Sinatra, Daniela. "Dionisio I e i Celti". Kokalos 42, 1996, 373-382.

Sordi, Marta. "Il IV e il III secolo da Dionigi I a Timoleonte (336 a.C.)". In Gabba-Vallet (edd.), 1979, 209-236.

Sordi, Marta. "I rapporti fra Dionigi I e Cartagine fra la pace del 405 e quella del 392". Aevum 54, 1980, 23-34.

Sordi, Marta. "I Galli in Apulia". Invigilata Lucernis 3-4, 1981-82, 5-11 = Ead. 1992, 133-139.

Sordi, Marta. "Timagene di Alessandria: uno storico ellenocentrico e filobarbaro". Aufstieg und Niedergang der Römischen Welt II 30.1, Berlin-New York, 1982, 775797.

Sordi, Marta. "Alessandro Magno, i Galli e Roma”. In Broilo (ed.), 1985, 207-214 = Ead. 1992, 121-131.

Sordi, Marta. La dynasteia in Occidente (studi su Dionigi I), Padova, Programma, 1992.

Sordi, Marta. "I due Dionigi, i Celti e gli Illiri". In Braccesi-Graciotti (edd.), 1999, 109$116=$ Ead. 2002, 577-585.

Sordi, Marta. “L'Europa di Filisto”. In Ead., ed., 2000a, 61-76.

Sordi Marta (ed.). Studi sull'Europa antica. Alessandria, dell'Orso, $2000 \mathrm{~b}$.

Sordi, Marta. Scritti di storia greca. Milano, Vita e Pensiero, 2002.

Struffolino, Stefano (ed.). Dinamiche culturali ed etniche nella Sicilia orientale dall'età classica all'epoca ellenistica. Atti del Convegno di Studi - Milano, 19-20 settembre 2013 = Aristonothos 4, Trento, Tangram 2014.

Sutton, Dana Ferrin. "Dithyramb as $\Delta \rho \tilde{\alpha} \mu \alpha$ : Philoxenus of Cythera's 'Cyclops or Galatea"'. Quaderni Urbinati di Cultura Classica 13, 1983, 37-43.

Szabó, Miklós. Les Celtes en Pannonie. Contribution à l'histoire de la civilisation celtique dans la cuvette des Karpates (Études d'Histoire et d'Archéologie 3). Paris, Presses de l'École Normale Supérieure, 1988.

Szabó, Miklós. “Celticité danubienne”. MÉFRA 119.1, 2007, 5-15.

Troiani, Sara. "Osservazioni sulla detorsio in comicum nel Ciclope di Filosseno fra tradizione omerica, critica metamusicale e satira politica". Lexis 36, 2018, 135-158.

Tsetskhladze, Gocha R. (ed.). Greek Colonisation. An account of Greek Colonies and Other Settlements Overseas, 2 (Mnemosyne Suppl. 193). Leiden-Boston, Brill, 2008.

Uggeri, Giovanni. "Dalla Sicilia all'Adriatico. Rotte marittime e vie terrestri nell'età dei due Dionigi (405-344)". In Bonacasa-Braccesi-De Miro (edd.), 2002, 295-320.

Urso, Gianpaolo. "Roma «città greca»: nota a Strabone V 3,5,232”. Aevum 75.1, 2001, 25-35.

Urso, Gianpaolo (ed.). Dall'Adriatico al Danubio. L'Illirico nell'età greca e romana. Atti del convegno internazionale, Cividale del Friuli 25-27 settembre 2003. Pisa, ETS, 2004.

Vaglio, Mariangela. "Filosseno e il Ciclope. Polifemo, i Galli e la propaganda di Dionigi di Siracusa". Hesperìa 14, Roma, L'Erma di Bretschneider, 2001, 171-177.

Vanotti, Gabriella. "Sulla cronologia della colonizzazione siracusana in Adriatico". Hesperia 2, Roma, L'Erma di Bretschneider, 1991, 107-110.

Vanotti, Gabriella. "L'archaiologia siciliana di Filisto". Hesperia 3, Roma, L'Erma di Bretschneider, 1993, 115-135.

Vanotti, Gabriella. “Alceta, Siracusa, Atene”. Hesperìa 7, Roma, L’Erma di Bretschneider, 1996, 77-90.

Vattuone, Riccardo. Sapienza d'Occidente. Il pensiero storico di Timeo di Tauromenio (Studî di Storia 4). Bologna, Pàtron, 1991. 
Vattuone, Riccardo. "Tradizioni locali e prospettive universali nella storiografia greca d'Occidente". In Bearzot-Id.-Ambaglio (edd.), 2001, 263-285.

Vian, Francis. Les origines de Thèbes. Cadmos et les Spartes (Études et commentaires 48). Paris, Klincksieck, 1963.

Waegeman, Maryse. "The Gecko, the Hoopoe ... and Lice". L'Antiquité Classique 53, 1984, 218-225.

Welch, Kathryn (ed.). Appian's Roman History. Empire and Civil War, Swansea, The Classical Press of Wales, 2015.

Westall, Richard. "The Relationship of Appian to Pollio: a Reconsideration". Analecta Romana Instituti Danici 38, 2013, 95-122.

Westall, Richard. "The Sources for the Civil Wars of Appian of Alexandria". In Welch (ed.), 2015, 125-167.

Wirth, Gerhard (ed.). Romanitas - Christianitas. Untersuchungen zur Geschichte und Literatur der römischen Kaiserzeit. Johannes Straub zum 70. Geburtstag am 18. Oktober 1982 gewidmet, Berlin, W. de Gruyter, 1982.

Woodhead, Geoffrey. “The 'Adriatic Empire' of Dionysius I of Syracuse”. Klio 52, 1970, 503-512.

Zaggio, Massimo - Mulas, Pier Luigi - Ceriana, Matteo. Giovanni Matteo Bottigella: cortigiano, uomo di lettere e committente d'arte: un percorso nella cultura lombarda di metà Quattrocento (Quaderni di Rinascimento 36). Firenze, Olschki, 1997. 